\title{
Comparação dos desfechos nos pacientes submetidos à amputação ou ao salvamento de membro como tratamento do osteossarcoma: uma revisão narrativa
}

\author{
Comparison of outcomes in patients submitted to the amputation or limb salvage as \\ treatment of osteosarcoma: a narrative review
}

\section{Comparación de resultados en pacientes presentados a amputación o recuperación del miembro como tratamiento de osteosarcoma: una revisión narrativa}

Bettina Geber ${ }^{1 *}$, Eduarda Abreu Figueiredo ${ }^{1}$, Isabela de Melo Assis ${ }^{1}$, Júlia Melo Coelho Lopes ${ }^{1}$, Luiza Fagundes Isolani ${ }^{1}$, Noele Maria Pereira e Queiroz ${ }^{1}$, Pedro Alcântara Miranda ${ }^{1}$, Reinaldo Andrade Neto ${ }^{1}$, Scheilla Torres de Oliveira ${ }^{2}$.

\section{RESUMO}

Objetivo: Realizar uma revisão narrativa comparando os desfechos, tais como impactos biopsicológicos e sobrevida, em pacientes submetidos à cirurgia de amputação ou de salvamentos de membros. Revisão bibliográfica: $\mathrm{O}$ osteossarcoma é uma neoplasia maligna comum em crianças e adolescentes, localizandose, principalmente, nas extremidades de ossos longos como a tíbia e o fêmur. Dos diversos tratamentos existentes, a amputação e a salvação do membro carecem de estudos comparando seus desfechos, impactos físicos e psicológicos e suas sobrevidas. Esses estudos conseguiriam auxiliar os médicos na escolha do tratamento mais adequado e com melhores resultados para cada paciente. Considerações finais: $O$ salvamento do membro apresenta melhor sobrevida sem aumentar o risco de recorrência local. No entanto, os pacientes que amputaram o membro obtiveram um ganho em bem-estar físico e psicológico quando comparados aos pacientes que salvaram o membro. Logo, este estudo afirma a necessidade de individualização do tratamento em cada caso, considerando os riscos, os benefícios e os impactos para cada paciente.

Palavras-chave: Osteossarcoma, Pediatria, Amputação, Salvamento de membro.

\begin{abstract}
Objective: Perform a narrative review comparing outcomes, such as biopsychological impacts and survival, in patients undergoing amputation or limb salvage surgery. Bibliographic review: Osteosarcoma is a common malignant neoplasm in children and adolescents, located mainly on the ends of long bones such as the tibia and femur. Of the various existing treatments, amputation and salvation of the limb lack studies comparing its outcomes, physical and psychological impacts and its survival. These studies would be able to assist doctors in choosing the most appropriate treatment and with the best results for each patient. Final considerations: Limb salvage has better survival without increasing the risk of local recurrence. However, patients who amputated the limb achieved a gain in physical and psychological well-being when compared to patients who saved the limb. Therefore, this study affirms the need for individualization of treatment in each case, considering the risks, benefits and impacts for each patient.
\end{abstract}

Keywords: Osteosarcoma, Pediatrics, Amputation, Limb salvage.

${ }^{1}$ Pontifícia Universidade Católica de Minas Gerais (PUC-MG), Belo Horizonte - MG.

${ }^{*}$ E-mail: be.g6199@gmail.com

${ }^{2}$ Santa Casa de Belo Horizonte, Belo Horizonte - MG.

SUBMETIDO EM: 7/2020

ACEITO EM: 8/2020

PUBLICADO EM: 11/2020 


\section{RESUMEN}

Objetivo: Realizar una revisión narrativa comparando los resultados, como los impactos biopsicológicos y la supervivencia, en pacientes sometidos a una amputación o cirugía de rescate de extremidades. Revisión bibliográfica: El osteosarcoma es una neoplasia maligna frecuente en niños y adolescentes, localizada principalmente en los extremos de huesos largos como la tibia y el fémur. De los diversos tratamientos existentes, la amputación y la salvación de la extremidad carecen de estudios que comparen sus resultados, impactos físicos y psicológicos y su supervivencia. Estos estudios podrían ayudar a los médicos a elegir el tratamiento más adecuado y con los mejores resultados para cada paciente. Consideraciones finales: Recuperación del miembro tiene una mejor supervivencia sin aumentar el riesgo de recurrencia local. Sin embargo, los pacientes que amputaron la extremidad lograron un aumento en el bienestar físico y psicológico en comparación con los pacientes que salvaron la extremidad. Por lo tanto, este estudio afirma la necesidad de individualizar el tratamiento en cada caso, considerando los riesgos, beneficios e impactos para cada paciente.

Palabras clave: Osteosarcoma, Pediatría, Amputación, Recuperación del miembro.

\section{INTRODUÇÃO}

O osteossarcoma é uma neoplasia maligna em que há um distúrbio de diferenciação óssea de origem mesenquimal. Particularmente é mais comum em crianças e adolescentes, sendo frequente também em adultos com 40 anos ou mais. Alguns fatores de risco estão relacionados com a doença, como a idade, sexo, raça, altura, genética e anormalidades ósseas congênitas (YANG Y, et al., 2018).

Esse câncer é comumente encontrado na extremidade de ossos longos, como a região distal do fêmur ou a região proximal da tíbia (CEPEDA MLA, et al., 2017). Apresentando uma predileção pelas metáfises de ossos longos, apresenta-se no fêmur distal em $43 \%$ dos casos, comparadas $23 \%$ na tíbia proximal e $10 \%$ no úmero (ISAKOFF MS, et al., 2015). A etiologia do osteossarcoma é desconhecida, mas os indivíduos que apresentam mutações nos genes que codificam as proteínas do retinoblastoma (Kb) e da p53 (TP53) têm maior risco de desenvolver esse tumor (LISENDA L, et al., 2017).

De acordo com Suárez A, et al. (2017), é a neoplasia óssea maligna mais comum em crianças e adolescentes, representando cerca de $2,5 \%$ de todos os cânceres em crianças com idade inferior a 15 anos e 4,2\% em adolescentes entre 15 e 19 anos. Possui uma incidência anual aproximada de 3,5 casos/milhão nos indivíduos menores de 15 anos e de 8,8 casos/milhão em pessoas de 15 a 19 anos.

Segundo Balmant N, et al. (2019), em análises epidemiológicas realizadas com amostras brasileiras, osteossarcomas correspondem a $66 \%$ dos tumores ósseos de membros inferiores, 10\% dos membros superiores e 3\% dos ossos pélvicos. Em meio aos subtipos morfológicos de tumores ósseos, os osteossarcomas apresentaram-se mais frequentes, quantificados em $47,3 \%$ dos casos.

Já em relação à incidência entre os sexos, foram observados 2,14 casos da doença a cada milhão de crianças do sexo masculino, comparados a 3,12 casos a cada milhão de crianças do sexo feminino. $O$ pico de incidência de osteossarcoma foi observado entre 10 a 14 anos nas meninas, sendo mais tardio entre os meninos, entre 15 a 19 anos.

O tratamento do osteossarcoma evoluiu ao longo da segunda metade do século XX, aumentando significativamente a taxa de sobrevida dos doentes. Até 1950, as opções terapêuticas eram a ressecção cirúrgica, mais precisamente a amputação, a radiação adjuvante ou a quimioterapia. Com esse tratamento os indivíduos tinham sobrevivência de $5 \%$, e uma taxa de sobrevida em 5 anos de apenas $22 \%$. Ainda, $80 \%$ dos pacientes diagnosticados com esse tumor tinham indícios de micrometástase, ou seja, apresentavam recaídas mesmo com a ressecção do tumor pela amputação (YANG Y, et al., 2018; SUÁREZ A, et al., 2017; LISENDA L, et al., 2017). 
A partir da década de 1970, a medicina avançou no setor de drogas quimioterápicas com resultados eficazes, propiciando a implementação da quimioterapia neoadjuvante, a qual provoca necrose tumoral e, consequentemente, possibilita uma excisão da neoplasia com a preservação do membro, o que facilita a recuperação do paciente, além de tratar micrometástases. Com isso, a taxa de sobrevida em 5 anos passou de 22\% para 66-82\% (YANG Y, et al., 2018; SUÁREZ A, et al., 2017; LISENDA L, et al., 2017).

$\mathrm{Na}$ década presente admite-se que a cirurgia de salvamento de membro seja indicada para os tumores localizados, ao passo que a amputação seja empregada para os casos de osteossarcoma de alta malignidade, dentre outros critérios de elegibilidade que serão abordados posteriormente (ANDERSON ME, 2016). Somadas às alternativas citadas, as modalidades biofísicas de tratamento têm tomado cada vez maior espaço no combate ao osteossarcoma, proporcionando resultados favoráveis aos pacientes (CARINA V, et al., 2019).

Apesar de toda evolução no tratamento do osteossarcoma e aumento de sobrevida dos pacientes ao longo dos últimos 50 anos, problematiza-se o fato de que esse progresso no manejo da doença não seja a realidade dos países em desenvolvimento, devido à escassez de recursos, tais como profissionais, equipamentos e esquemas quimioterápicos (LISENDA L, et al., 2017).

Com isso, estudos de revisão avaliando os impactos físicos e psicológicos dos tratamentos desse câncer na vida dos pacientes, além da sobrevida frente ao tipo de terapêutica proposta, devem ser incentivados, a fim de nortear a melhor abordagem considerando os recursos disponíveis e a qualidade de vida do paciente após a intervenção. Dessa forma, o presente artigo tem o objetivo de comparar os desfechos, tais como impactos biopsicológicos e sobrevida, dos pacientes com osteossarcoma submetidos à cirurgia de salvamento de membro em relação aqueles em que foi realizada a cirurgia de amputação.

\section{REVISÃO BIBLIOGRÁFICA}

\section{Desfechos do Osteossarcoma}

As principais consequências do Osteossarcoma se relacionam aos efeitos sintomáticos na qualidade de vida dos pacientes, provocadas pela dor, edema/rigidez articular e traumas patogênicos em áreas de articulação, os quais impactam na limitação do movimento e no comprometimento da marcha com interrupção das atividades diárias (SILVA TMR, et al., 2017).

Diante da sintomatologia de dor constante, da diminuição da capacidade de movimento e, somado ao aspecto estético do contexto patológico, o estado emocional e psicológico dos pacientes e familiares podem ser afetados de forma significativa, induzindo quadros de ansiedade, tristeza, exclusão e distanciamento social e sentimento de impotência. Sintomas respiratórios, apesar de raros, podem se manifestar em pacientes com Osteossarcoma, sendo relacionados ao surgimento de metástases pulmonares (GIL S, et al., 2019).

As taxas de sobrevida global e de sobrevida livre de eventos são os índices mais utilizados como método prognóstico e podem ser definidas como porcentagem de paciente vivos em um determinado período de tempo após o diagnóstico ou início do tratamento e o período de tempo após um tratamento durante o qual o paciente permanece sem apresentar complicações e eventos que a terapia almeja evitar, respectivamente (CHEN W e LIN Y, 2019).

Uma relação pode ser estabelecida entre o tamanho do tumor, a localização, a evolução, sexo, histologia, presença de metástase, a forma de tratamento e a resistência medicamentosa na definição das taxas de mortalidade e sobrevida dos pacientes. Estudos têm demonstrado que o grau de Huvos (I, II, III e IV) - que avalia a percentagem de necrose tumoral após quimioterapia neoadjuvante, o tamanho do tumor $(<15$ e $>15$ $\mathrm{cm}$ ), a presença de metástase ao diagnóstico e o tratamento cirúrgico usado (radical e salvador) foram definidas como variáveis significativas para determinação das sobrevidas.

Os fatores prognósticos desfavoráveis observados foram sexo masculino, metástase ao diagnóstico, tamanho do tumor maior do que $15 \mathrm{~cm}$ e tratamento cirúrgico radical, sendo a presença de metástase como fator de pior prognóstico e resposta quimioterápica como fator mais importante para melhores desfechos 
(BICHOP M, et al., 2016; STOKKE J, et al., 2015). Outras variáveis de prognóstico encontradas relacionadas a uma maior mortalidade no osteossarcoma incluem idade avançada, pacientes negros, localização em membros superiores ou no esqueleto axial, tipo histológico tumoral, principalmente Osteossarcoma de Paget, além de estadiamento tumoral (ZHENG W, et al., 2018; TRAVEN SA, et al., 2019).

\section{Amputação}

Por muitas décadas a opção terapêutica para osteossarcoma limitou-se à amputação de membros. Com a introdução do conhecimento sobre quimioterapia nesse cenário, houve uma melhora de sobrevida dos pacientes, em cerca de 20 a 65\% com os regimes multiagentes (QI L, et al., 2020). Isso fez com que a cirurgia poupadora de membros (CPM) ganhasse notoriedade, sendo usada na maioria dos casos e passando a ser o foco das estratégias cirúrgicas, o que foi possibilitado pelos avanços nas técnicas de imagem, além dos efeitos positivos da quimioterapia pré-operatória (GIL S, et al., 2019; HE X, et al., 2017).

De forma resumida, o estudo de Lin Qi, et al. (2020) observou que os principais candidatos para amputação de membros são pacientes com idade entre 41 e 60, sexo masculino, pelve como local primário, tumor de alto grau (III / IV), estágio de disseminação regional ou distante, tamanho do tumor $>92 \mathrm{~mm}$ e casos sem radioterapia. A ressecção cirúrgica completa, ainda é justificada quando salvamento de membros for contraindicado, em pacientes com resposta ineficaz à quimioterapia, fratura patológica ou em tumores com características clinicamente mais agressivas, que envolvem estruturas neurovasculares importantes ou são tão extensos, que mesmo com CPM o membro permaneceria não funcional (QI L, et al., 2020; TRAVEN SA, et al., 2019)

Pesquisas comparando a CPM e a amputação de membros, constataram uma melhor sobrevida em pacientes submetidos a primeira abordagem. Contudo, esse resultado ocorre possivelmente por um pior prognóstico pré-terapêutico dos pacientes que sofreram amputação, evidenciando um provável viés de seleção, o que dá margem para o questionamento se o aumento de sobrevida seria significativo e relacionado ao procedimento (TRAVEN SA, et al., 2019).

Levando isso em consideração, um estudo de Gil S et al. (2019) identificou que o alívio sintomático, seria o objetivo principal da intervenção cirúrgica, tanto na visão dos cirurgiões como dos pacientes, mesmo que não ocorresse prolongamento da sobrevida. A amputação torna-se, então, importante principalmente para os pacientes com baixa chance de sobrevivência, já que podem melhorar a capacidade de realizar atividades diárias e reduzir a dor, o que proporciona melhoria na vida emocional, sexual e social (GIL S, et al., 2019).

Ao optar pela realização de excisão cirúrgica completa, vários aspectos devem ser levados em consideração, como psicológicos, principalmente se tratando de crianças. Para isso, é necessária uma abordagem multidisciplinar objetivando uma assistência integral ao paciente. Sobre os impactos que tal abordagem poderia acarretar à qualidade de vida do paciente, no estudo de Gil S, et al. (2019) foi demonstrado que apesar da preocupação acerca das consequências emocionais que uma amputação pudesse causar, a maioria das crianças obtiveram um ganho em bem-estar psicológico, motivadas pela aprendizagem do uso de próteses e pela independência adquirida após o procedimento.

Além disso, a maioria dos pacientes apresentaram, dentro de alguns meses, uma melhora na deficiência física e um estilo de vida mais ativo após a excisão cirúrgica completa, levando em consideração que estes apresentavam um declínio progressivo da mobilidade antes do procedimento. No entanto, Qi L, et al. (2020) afirmam que, apesar dos benefícios apresentados, algumas limitações podem ser apontadas, como a frequente necessidade de margem cirúrgica ampla, devido a agressividade da neoplasia, o que reduz o tamanho do coto preservado. Com isso, pode haver dificuldade de adaptação de próteses que alcancem a função orgânica e psicossocial idealizada.

\section{Salvamento de membros}

Com os crescentes avanços da quimioterapia neoadjuvante, diagnósticos por imagem e materiais de reconstrução, as técnicas de salvamento de membros vêm ganhando destaque e tem se tornado a estratégia de maior destaque quando se fala de tratamento de osteossarcomas (SILVA R, et al., 2019). 
A ablação tumoral é uma técnica de salvamento de membros caracterizada pelo uso de métodos físicos ou químicos, que tem como objetivo a eliminação das células tumorais. Existem diversos tipos de ablação, dentre eles destacam-se a ablação por temperatura, que utiliza radiofrequência, micro-ondas, ultrassom de alta intensidade, laser e crioablação. Além da ablação por método químico, que engloba o uso de álcool anidro e ácido acético glacial. Esta técnica vem sendo utilizada cada vez mais para o tratamento de salvamento de membros em pacientes com osteossarcoma, demonstrando efeitos clínicos positivos.

A ablação por ultrassom focada em alta intensidade (HIFU) utiliza de energia de ultrassom focada no tecido tumoral alvo, levando este à morte por efeitos térmicos e de cavitação, ativando também o sistema imunológico antitumoral. O tempo médio de sobrevida dos pacientes que realizam esta modalidade terapêutica é de 68 meses, sendo a taxa de sobrevida em 5 anos avaliada em 71,4\%. O uso da HIFU em tumores ósseos malignos primários apresentou efeito geral em $84,6 \%$ e sobrevida em 5 anos de $38,5 \%$ dos casos. Em relação aos tumores ósseos metastáticos foi observado efeito geral em $75 \%$ dos pacientes, com sobrevida de 5 anos avaliada em $0 \%$. O uso desta técnica ainda demanda investigações acerca de sua eficácia (YANG Y, et al., 2018).

A ablação por microondas utiliza ondas de rádio, causando oscilação das moléculas de água e criando termogênese. Desta forma, acarreta em necrose de coagulação das células tumorais. Esta técnica é realizada após delimitação da área do tumor, preservando o tecido normal adjacente. Uma vez delimitada a área alvo, uma sequência de ablações é realizada visando inativar o tumor in situ. Em meio a 153 pacientes com osteossarcoma tratados com esta técnica, a sobrevida em 5 anos observada foi de $73,9 \%$, apresentando grande melhora da função do membro. Esta é uma nova modalidade terapêutica que se demonstrou eficaz, além de atuar aumentando a sensibilidade dos tumores à radiação e à quimioterapia, sendo avaliada como promissora à medida em que os avanços em pesquisas ocorrem.

A crioablação é constituída por ciclos de resfriamento rápido seguido de descongelamento lento. A partir de alterações microvasculares, hipóxia, isquemia e liberação de antígenos, as células tumorais sofrem danos, sendo levadas à morte imune. Em meio a 440 pacientes com tumores ósseos tratados com esta técnica foi avaliada excelente função dos membros em $84,5 \%$ dos pacientes, recorrência local em $8 \%$ e complicações em $6,3 \%$. A crioablação não apresenta nenhuma toxicidade e nenhuma atividade radioativa, possui efeito analgésico e pode evitar a ressecção cirúrgica temporária ou permanente. Entretanto, seu efeito terapêutico ainda precisa ser acompanhado a longo prazo (YANG Y, et al., 2018).

A aplicação do sistema de navegação assistida por computador (CANS) diz respeito a uma técnica cirúrgica digital que tem apresentado sucesso na prática clínica nos últimos anos. No tratamento de tumores ósseos a CANS apresenta vantagens em relação aos métodos tradicionais quanto ao planejamento cirúrgico e pré-operatório, ressecção óssea e implantação protética. Em uma observação de 3 pacientes com tumores ósseos tratados com a CANS os resultados se mostraram satisfatórios, não sendo relatadas recorrências ou metástases em uma média de 28 meses de seguimento. Em contrapartida a referida modalidade cirúrgica também apresenta desvantagens, exemplificadas pelas instalações pesadas, complexidade da operação e necessidade de profissionais devidamente experientes (YANG Y, et al., 2018).

A prevenção de infecção após as cirurgias de resgate de membros no osteossarcoma apresenta importância expressiva, visto que esta constitui a complicação mais importante na recuperação dos pacientes, atingindo até $30 \%$ dos casos. Em 51 casos de reconstrução com prótese banhada a ouro a taxa de infecção pós-operatória apresentou-se em 5,9\%, comparadas a 17,6\% em 74 casos de reconstrução com próteses de liga de titânio. Neste último grupo, 38,5\% dos pacientes apresentaram necessidade de amputação pela infecção periprotética. Algumas modalidades protéticas demonstraram resultados mais satisfatórios, como a prótese banhada a prata, a prótese de liga de titânio revestida com iodo e próteses revestidas com antibióticos (TRAVEN SA, et al., 2019).

O tratamento por cirurgia de salvamento de membros pode gerar diversas complicações, como infecção por implante, afrouxamento e quebra, diferença no comprimento dos membros, no entanto, as técnicas vêm sendo cada vez mais aprimoradas para que o uso desse tratamento seja cada vez mais eficiente, de forma a 
melhorar ainda mais a qualidade de vida dos pacientes com osteossarcoma. Mesmo com esse avanço das técnicas, é necessário acompanhar a eficácia desses tratamentos a longo prazo, para que continuem sendo melhorados e para se tornarem cada vez mais eficazes (QI L, et al., 2020).

\section{Sobrevida e mortalidade}

Comparativamente, a maior parte dos estudos analisados mostra uma maior sobrevida nos pacientes que foram submetidos ao salvamento do membro, em relação aos que foram amputados. O estudo de Traven AS, et al. (2019) obteve como resultados um tempo de sobrevida médio de 55 meses em todos os pacientes diagnosticados com osteossarcoma, a partir do momento do diagnóstico, sendo que os submetidos ao salvamento do membro tiveram uma sobrevida maior, de 66 meses, e os pacientes que sofreram amputação, 45 meses de sobrevida em média. As taxas de mortalidade relacionada ao câncer, neste estudo, acompanham essa tendência, com uma maior chance nos pacientes que foram amputados em comparação aos que tiveram seus membros salvos.

Qi L, et al. (2020) apresentam comparação estatisticamente significante em relação à sobrevivência relacionada ao câncer, cause-specific survival (CSS) e a sobrevivência geral, overall survival (OS) nos dois tipos de intervenção.

Em relação ao tratamento de salvamento do membro, a sobrevivência em 3 e 5 anos relacionada ao câncer foi, respectivamente, de $74,5 \%$ e $66,2 \%$, e a taxa de sobrevivência geral foi de $78,9 \%$ e $70,9 \%$. Na amputação, as taxas de sobrevivência em 3 e 5 anos relacionadas ao câncer foram de 67,4\% e 59,4\%, e a sobrevivência geral, $64,5 \%$ e $55,3 \%$.

O tratamento com preservação do membro se mostrou superior, em relação às duas análises da sobrevivência, principalmente em pacientes entre 21 e 40 anos. Faisham Wl, et al. (2015) também demonstraram uma menor taxa de mortalidade em 2 e 5 anos nos pacientes que foram tratados com 0 salvamento do membro em comparação ao grupo de amputação, sendo o procedimento cirúrgico realizado após 3 ciclos de quimioterapia.

Em relação a recorrência do osteossarcoma, em pacientes tratados cirurgicamente com margens adequadas, não houve diferença significativa entre as taxas de recorrência no salvamento do membro e amputação (QI L, et al., 2020; TRAVEN SA, et al., 2019; HAN K, et al., 2017).

Segundo Deng ZP, et al. (2015), em pacientes que sofreram fraturas patológicas e foram submetidos ao procedimento de salvamento de membro a taxa de recorrência foi maior, no entanto, não impactou com a sobrevida geral dos pacientes em comparação aos que foram amputados. A ocorrência de fraturas patológicas não aumentou de forma estatisticamente significante a mortalidade do osteossarcoma.

\section{CONSIDERAÇÕES FINAIS}

Dado o exposto, apesar da evolução no tratamento do osteossarcoma, ainda existem questionamentos acerca da melhor abordagem, sobretudo entre o salvamento do membro ou sua amputação. O presente artigo demonstra que a sobrevida nos pacientes que foram submetidos ao salvamento do membro foi considerada maior quando comparada a sobrevida dos pacientes que amputaram, possivelmente devido ao viés de seleção. Em relação a recorrência do osteossarcoma, não houveram significativas diferenças entre os dois grupos. No entanto, foi apresentado que os pacientes que amputaram o membro obtiveram um ganho em bem-estar físico e psicológico quando comparados aos pacientes que salvaram o membro. Em vista disso, o artigo afirma a necessidade de individualização do tratamento em cada caso, considerando os riscos, os benefícios e os impactos para cada paciente. Ainda, este estudo sugere mais pesquisas na área para eliminar o viés de seleção relacionado ao pior prognóstico pré-terapêutico dos pacientes que sofreram amputação, a fim de obtermos uma defesa mais definida do melhor tratamento tendo em vista os impactos no indivíduo.

\section{AGRADECIMENTOS E FINANCIAMENTO}

Os autores agradecem o apoio da Liga Acadêmica de Oncologia (LAONCO) e do Diretório Acadêmico da Pontifícia Universidade Católica de Minas Gerais. Este artigo não dispôs de financiamento. 


\section{REFERÊNCIAS}

1. ANDERSON ME. Update on survival in osteosarcoma. Orthopedic Clinics of North America, 2016; 47(1): $283-292$.

2. BALMANT NV, et al. Incidence and mortality of bone cancer among children, adolescents and young adults of Brazil. Clinics, 2019. 74: e858.

3. BICHOP M, et al. Future Directions in the Treatment of Osteossarcoma. Current Opinion in Pediatrics, 2016; 28(1): 2633.

4. CARINA V, et al. Adjuvant Biophysical Therapies in Osteosarcoma. Cancers (Basel), 2019. 11:11.

5. CEPEDA MLA, et al. Osteosarcoma telangiectásico en un lactante. Boletín Médico del Hospital Infantil de México, 2017; 74(1): 60-64.

6. CHEN W, LIN Y. Nomograms Predicting Overall Survival and Cancer-Specific Survival in Osteosarcoma Patients (STROBE). Medicine (Baltimore), 2019; 98(26): e16141.

7. DENG ZP, et al. The Surgical Treatment and Outcome of Nonmetastatic Extremity Osteosarcoma with Pathological Fractures. Chinese Medical Journal, 2015; 128(19): 2605-2608.

8. FAISHAM WI, et al. Prognostic Factors and Survival Rate of Osteosarcoma: A Single-Institution Study. Asia-Pacific Journal of Clinical Oncology, 2017; 13(2): e104-e110.

9. GIL S, et al. Role of Amputation in Improving Mobility, Pain Outcomes, and Emotional and Psychological Well-Being in Children With Metastatic Osteosarcoma. American Journal of Hospice and Palliative Medicine, 2019; 36(2): 105-110.

10. HAN K, et al. Is Limb Salvage With Microwave-induced Hyperthermia Better Than Amputation for Osteosarcoma of the Distal Tibia?. Clinical Orthopaedics and Related Research, 2017; 475(6): 1668-1677.

11. HE X, et al. A meta-analysis of randomized control trials of surgical methods with osteosarcoma outcomes. Journal of Orthopaedic Surgery and Research, 2017; (12)5.

12. ISAKOFF MS, et al. Osteosarcoma: Current Treatment and a Collaborative Pathway to Success. Journal of Clinical Oncology, 2015; 33:3029-3035.

13. LISENDA L, et al. Osteosarcoma outcomes at a South African tertiary hospital. South Africa Medical Journal, 2017; 107(9): 754-757.

14. QI L, et al. Predictors and Survival of Patients with Osteosarcoma After Limb Salvage versus Amputation: A PopulationBased Analysis with Propensity Score Matching. World Journal of Surgery, 2020; 44: 2201-2210.

15. SILVA TMR, et al. Itinerário terapêutico de adolescentes com osteossarcoma: implicações para o diagnóstico precoce. Revista Mineira de Enfermagem, 2017; 21: e1028.

16. SILVA R, et al. Quality of life of patients with sarcoma after conservative surgery or amputation of limbs. Revista Acta Ortopédica Brasileira, 2019; 27(5): 276-80.

17. STOKKE J, et al. Systematic Review and Meta-Analysis of Objective and Subjective Quality of Life Among Pediatric, Adolescent, and Young Adult Bone Tumor Survivors. Pediatric Blood Cancer, 2015; 62(9): 1616-1629.

18. SUÁREZ A, et al. Resultados del tratamiento de osteosarcoma convencional de alto grado en niños y adolescentes: análisis de supervivencia de una cohorte tratada sin metotrexato. The Revista Colombiana de Cancerología, 2017; 21(2): 86-94.

19. TRAVEN SA, et al. A propensity-score matched analysis of limb salvage vs amputation for osteosarcoma. Journal of Surgical Oncology, 2019; 120(7): 1252-1258.

20. YANG Y, et al. Advances in limb salvage treatment of osteosarcoma. Journal of Bone Oncology, 2018; 10: 36-40.

21. ZHENG W, et al. Nomogram application to predict overall and cancer-specific survival in osteosarcoma. Cancer Management and Research, 2018; 10: 5439-5450. 